\title{
High-dose immunoglobulin pulse therapy and risk of Covid19 infection
}

\author{
Valeria Prada ${ }^{1}$ (1) Luana Benedetti $^{1,2} \cdot$ Dario Cocito $^{3} \cdot$ Chiara Briani $^{4} \cdot$ Eduardo Nobile Orazio $^{5} \cdot$ Francesca Gallia $^{5}$. \\ Giovanni Antonini $^{6}$. Fiore Manganelli ${ }^{7}$. Gian Maria Fabrizi ${ }^{8} \cdot$ Francesco Germano $^{1}$ Marina Grandis ${ }^{1,2}$. \\ Angelo Schenone ${ }^{1,2}$
}

Received: 26 June 2020 / Revised: 5 August 2020 / Accepted: 6 August 2020 / Published online: 10 August 2020

c) Springer-Verlag GmbH Germany, part of Springer Nature 2020

\section{Dear Sirs,}

COVID-19 emerged as a novel disease at the beginning of 2020 and leads to acute respiratory syndrome. There is no vaccine or specific therapy available. Interestingly, therapy with hyperimmune plasma from recovered COVID19 patients has been suggested as a possible therapeutic approach [1]. Intravenous immunoglobulins (IVIg) may harbour some protective effect, even in the absence of direct contact by donors with the infective agent [2].

We focused our study on patients affected by immunemediated neuromuscular diseases [3]. The majority of them are chronically treated with a high dosage of IVIg or subcutaneous (SCIg) immunoglobulins, giving us the opportunity of evaluating a large number of subjects and compare the

Valeria Prada

valeria.prada@gmail.com

1 Department of Neurosciences, Rehabilitation, Ophtalmology, Genetics and Maternal/Child Sciences, University of Genova, Largo Daneo 3, 16132 Genova, Italy

2 Department of Neurology, IRCCS Policlinico San Martino, Genova, Italy

3 Istituti Clinici Scientifici Maugeri, Torino, Italy

4 Department of Neurosciences, University of Padova, Padova, Italy

5 Department of Medical Biotechnology and Translational Medicine, Neuromuscular and Neuroimmunology Service, Humanitas Clinical and Research Institute, Milan University, Milan, Italy

6 Department of Neurosciences, Mental Health and Sensory Organs, University of "La Sapienza", Rome, Italy

7 Department of Neurosciences, Reproductive and Odontostomatological Sciences, University of Naples "Federico II", Naples, Italy

8 Department of Neurosciences, Biomedicine and Movement Sciences, University of Verona, Verona, Italy results with a control group represented by patients with the same diseases following other immunomodulatory therapies.

IVIg produced before the $\mathrm{H} 1 \mathrm{~N} 1$ influenza pandemic, avoid the lung replication of the virus, and protect against mortality because of a cross-reaction of the antibody to this virus [2]. Hemagglutinin and neutralising antibodies against pandemic influenza have been found in IVIg and they have a protective effect from $\mathrm{H} 1 \mathrm{~N} 1$ infection. IVIg effectively inhibit the replication of $\mathrm{HCoV}-\mathrm{NL} 63$, a coronavirus which leads to acute respiratory syndrome in adults and to Kawasaki disease in children, similar to SARS-CoV-2, so that commercially available immunoglobulins may represent a potential therapy for treating acute respiratory illness [4]. Moreover, pre-pandemic IVIg can provide protection by a mechanism of antibodies cross-reaction induced by repeated exposure to seasonal H1N1 or coronaviruses, and hyperimmune post-pandemic IVIg may be more effective in protecting the host, as demonstrated in mice [5].

To address the potential protective effect of IVIg against COVID-19 infection, even if obtained before the pandemic, we conducted, through the CIDP Italian Association, an online anonymous questionnaire among patients affected by immune-mediated neuromuscular diseases, from 4 to 28 April. In this population, we assessed possible COVID-19 infection and the effects of other immunosuppressive therapies. We chose an online questionnaire due to the impossibility of reaching all the patients because of the lockdown and in order to collect a high number of subjects in a fast way. This method has obviously different limitations, first of all, we could contact only the patients afferent to the association or to outpatients' hospital facilities and thus only patients with an ongoing therapy. Therefore, we could not recruit patients with previous diseases and without therapy. Anyway, considering the limitation of the lockdown the online questionnaire was the only way to conduct a survey. 213 patients participated in the survey, but we had to exclude two 
incomplete questionnaires. The majority of patients suffered from chronic inflammatory demyelinating polyradiculoneuropathy (CIDP, $73.9 \% ; n$ : 156), followed by other immunemediated neuromuscular diseases. In this population, $46 \%$ did not report any comorbidities, $35.5 \%$ had one comorbidity and only a minority had more than one comorbidity (14.5\%; Table 1). 165 patients were under monthly IVIg treatment or SCIg. The others ( $n: 46)$ had different therapies, such as immunomodulatory drugs or no therapy (Table 1 ). Four patients contracted COVID-19. We excluded patients with general symptoms but without a laboratory confirmation to exclude some evaluation mistakes or biases due to misjudgement by the patients ( $n$ : 16). Only one was under IVIg therapy and the other three patients were on steroids $(n: 2)$ or no therapy ( $n: 1$; Table 1$)$. Comparing these data, where patients with no IVIg therapy can be considered a control group, with a Chi-square test and a Yates correction, the difference between these two groups is significant (Chi square: 3.887; degrees of freedom: $1, p<0.05$; Table 1). Finally, we observed that the incidence of COVID-19 infection in our selected population is $2 \%$. These data suggest that patients undergoing IVIg or SCIg chronic treatments may have a reduced risk of contracting COVID-19 infection compared to patients receiving other immune therapies or no therapy.

In conclusion, we can hypothesise that, as already demonstrated, chronic immunoglobulin therapy may protect or reduce the risk of contracting infections, including COVID19. More studies based on medical recording and with a stratification of patients based on type of disease and on degree of severity, are needed to confirm our conclusions, which could possibly be conducted when the COVID-19 emergency ends and access to clinical data and visits will be available again.

Table 1 Resume of data collected

\begin{tabular}{|c|c|c|c|c|}
\hline \multicolumn{2}{|l|}{ General information } & \multicolumn{3}{|c|}{ Comorbidities-number (\%) } \\
\hline Total of patients (number) & 213 & \multicolumn{2}{|c|}{ Total } & 211 \\
\hline Excluded & 2 & & No comorbidities & $97(46)$ \\
\hline Patients included & 211 & & One comorbidity & $75(35.5)$ \\
\hline Female-number (\%) & $84(39.8)$ & & More than one comorbidity & $39(14.5)$ \\
\hline Mean age (range)-years & $39-79(52.3)$ & & & \\
\hline \multicolumn{2}{|l|}{ Diseases-number (\%) } & \multicolumn{3}{|c|}{ Type of comorbidities-number (\%) } \\
\hline Total & $211(100)$ & \multicolumn{2}{|c|}{ Total } & $151(100)$ \\
\hline CIDP & $156(73.9)$ & \multicolumn{2}{|c|}{ Respiratory } & $23(15.2)$ \\
\hline GBS & $9(4.3)$ & \multicolumn{2}{|c|}{ Cardiovascular } & $45(29.8)$ \\
\hline MMN & 31 (14.7) & \multicolumn{2}{|c|}{ Dysimmune } & $17(11.3)$ \\
\hline Myasthenia & $6(2.8)$ & \multicolumn{2}{|c|}{ Neurologic } & $9(6)$ \\
\hline Anti-MAG antibody neuropathy & $7(3.3)$ & \multicolumn{2}{|c|}{ Metabolic } & $21(13.9)$ \\
\hline Vasculitis & $1(0.5)$ & \multicolumn{2}{|c|}{ Tumours } & $5(3.3)$ \\
\hline Parsonage Turner & $1(0.5)$ & \multicolumn{2}{|c|}{ Others } & $31(20.5)$ \\
\hline \multicolumn{2}{|l|}{ Therapy-number (\%) } & Therapy (total) & Negative & Positive \\
\hline Total & \multicolumn{4}{|l|}{$211(100)$} \\
\hline IVIg & $104(49.3)$ & IVIg (95) & $90.4 \%(94)$ & $1 \%(1)$ \\
\hline SCIg & $55(26.1)$ & SCIg (52) & $94.5 \%(52)$ & \\
\hline Steroids & $17(8)$ & Steroids (17) & $88.2 \%(15)$ & $11.8 \%(2)$ \\
\hline Other therapies & $9(4.3)$ & Other therapies (7) & $77.8 \%(7)$ & \\
\hline No therapy & $7(3.3)$ & No therapy (7) & $85.7 \%(6)$ & $14.3 \%(1)$ \\
\hline Rituximab & $6(2.8)$ & Rituximab (5) & $83.3 \%(5)$ & \\
\hline IVIg + SCIg & $5(2.4)$ & IVIg + SCIg (5) & $100 \%(5)$ & \\
\hline Azathioprine & $4(1.9)$ & Azathioprine (4) & $100 \%(4)$ & \\
\hline Plasmapheresis & $3(1.4)$ & Plasmapheresis (2) & $66.7 \%(2)$ & \\
\hline Cyclophosphamide & $1(0.5)$ & Cyclophosphamide (1) & $100 \%(1)$ & \\
\hline & Negative & Positive & Chi square, df & $P$ value \\
\hline IVIg, SCIg (152) & $92.1 \%(151)$ & $0.6 \%(1)$ & $3.887,1$ & $* 0.0487$ \\
\hline Other therapies (43) & $85.1 \%(40)$ & $6.4 \%(3)$ & & \\
\hline
\end{tabular}


Funding No funding was received for this work.

\section{Compliance with ethical standards}

Conflict of interest We wish to confirm that there are no known conflicts of interest associated with this publication and there has been no significant financial support for this work that could have influenced its outcome.

Ethics approval Data collected were in accordance with the ethical standards of the responsible committee on human experimentation and with the Helsinki Declaration of 1975, as revised in 2000. Informed consent was obtained by specific questions of the questionnaire from all patients for being included in the study.

\section{References}

1. Jawhara S (2020) Could intravenous immunoglobulin collected from recovered coronavirus patients protect against covid-19 and strengthen the immune system of new patients? Int J Mol Sci 21(7):2272

2. Rockman S, Lowther S, Camuglia $S$ et al (2017) Intravenous immunoglobulin protects against severe pandemic influenza infection. EBioMedicine. https://doi.org/10.1016/j.ebiom.2017.04.010

3. Haberman R, Axelrad J, Chen A et al (2020) Covid-19 in immunemediated inflammatory diseases - case series from New York. N Engl J Med. https://doi.org/10.1056/NEJMc2009567

4. Pyrc K, Bosch BJ, Berkhout B et al (2006) Inhibition of human coronavirus NL63 infection at early stages of the replication cycle. Antimicrob Agents Chemother. https://doi.org/10.1128/ AAC.01598-05

5. Hohenadl C, Wodal W, Kerschbaum A et al (2014) Hyperimmune intravenous immunoglobulin containing high titers of pandemic H1N1 hemagglutinin and neuraminidase antibodies provides dose-dependent protection against lethal virus challenge in SCID mice. Virol J. https://doi.org/10.1186/1743-422X-11-70 